\title{
Study on the Curriculum Design for Foreign Language Teaching from the Perspective of "Capabilities-based Planning"
}

\author{
Ma Xiaolei ${ }^{1, \mathrm{a}}, \mathrm{Hu}$ Xiaotang $^{1, \mathrm{~b}}$, Chen Yingfang ${ }^{1, \mathrm{c}}$, Li Ping ${ }^{1, \mathrm{~d}}$

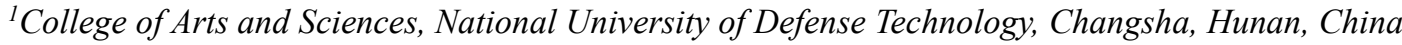 \\ amaxiaolei@nudt.edu.cn \\ ${ }^{b}$ huxiaotang2015@nudt.edu.cn \\ c4531768@qq.com \\ d1156542338@qq.com
}

\begin{abstract}
The insubstantial guidance of talent cultivation objectives and the insufficient support of teaching systems pose current constraints on foreign language teaching for Chinese colleges and universities. To overcome these constraints, curriculum designers need to systematically deliberate on talent cultivation objectives, and then to scientifically integrate the objectives into all aspects of the curriculum. Similarly, under the framework of the US military's "Capabilities-based Planning (CBP)", talent cultivation objectives, instead of being treated in an isolated way, serve as a main thread linking up goals, content, methods, assessment and other elements of teaching. In this sense, CBP can create more access to educational planning. By showcasing the curriculum design for Master of Interpreting and Translation, this study aimed to explore the applicability of CBP to education.
\end{abstract}

Keywords: Capabilities-based Planning, curriculum design, foreign language

\section{“基于能力的规划”视角下外语课程教学方案设计研 究} 马晓雷 ${ }^{1,}$ a 胡小棠 ${ }^{1,}{ }^{b}$ 陈颖芳 ${ }^{1, \mathrm{c}}$ 李平 ${ }^{1, \mathrm{~d}}$

${ }^{1}$ 国防科技大学文理学院, 长沙, 湖南, 中国

amaxiaolei@nudt.edu.cn

${ }^{b}$ huxiaotang2015@nudt.edu.cn

c4531768@qq.com

d1156542338@qq.com

\section{摘要}

人才培养目标统领作用不实、教学体系支撑不足是当前高校外语教学普遍面临的困境。要解决这些难题, 就需 要教学设计者以系统化的思维方式审视人才培养目标, 并以科学的规划方法将培养目标贯穿于教学的各个环 节。美军 “基于能力的规划” 框架，可以为教育领域的规划设计提供参考。该框架不是孤立地看待人才培养目 标, 而是以其为主线将教学目标、内容、方法和评估等要素纵向衔接、垂直贯通。以翻译专业硕士教学方案设 计为例，本研究拟探讨 “基于能力的规划” 框架在教育领域的应用价值。

关链词: 基于能力的规划，课程设计，外语

\section{1. 前言}

高等教育的主要职能是培养人才、服务于经济社
会发展。2016 年 12 月 7 日, 在全国高校思想政治工 作会议上, 习近平主席指出: “要提高我国高等教育 发展水平, 增强国家核心竞争力……必须牢牢抓住全 
面提高人才培养能力这个核心点, 并以此来带动高校 其他工作。”国务院印发的《国家中长期教育改革和 发展纲要》也提到: “高等教育承担着培养高级专门 人才、发展科学技术文化、促进社会主义现代化建设 的重大任务……提高人才培养质量。牢固确立人才培 养在高校工作中的中心地位, 着力培养信念执著、品 德优良、知识丰富、本领过硬的高素质专门人才和拔 尖创新人才。”虽然党中央、国务院和习近平主席对 高等教育人才培养工作十分重视, 也对此提出了明确 要求, 但高等教育的现状却不容乐观。当代著名教育 家潘禁元 (潘禁元、李国强, 2016: 6) 认为, 大多数 高等学校沿着传统大学单一的道路走, 脱离社会所需 要, “导致高校同质化、学生就业难”。高校教学改 革的首要任务应为结合社会经济发展需求, 明确定位 人才能力目标。

而且, 能力目标需要融入人才培养模式的各个环 节才能真正有效。最早论及人才培养模式要素的是刘 明浚（1993），他认为其中包括 “课程体系、教育途 径、教学方法、教学手段、教学组织手段等”。周远 清 (1998) 提出, 人才培养模式的要素是培养目标、 培养规格和基本培养方式。刀维国（2008）把人才培 养模式梳理为五个方面: 理论依据、教学目标、操作 程序、实现条件、教学评价。祝家贵 (2015) 将人才 培养模式归纳为培养目标和规格, 为实现培养目标和 规格的整个教育过程, 为实现这一过程的一整套管理 和评估制度, 以及与之相匹配的教学方式、方法和手 段等四个环节。这些观点本质上趋同, 大都涉及 “培 养目标和规格”、“教育过程”、“教学模式、方法 与手段”、“管理评估制度” 等环节。“培养目标和 规格”最为重要。根据“泰勒原理 (the Tyler Rationale)” (1949), 教育目标是选择教育经验、组织教学内容 以及评价教学结果的准则, 即教育目标在各个环节都 起主导作用。只有当 “培养目标和规格” 落实到 “教 育过程”、“管理评估制度”、“教学模式、方法与 手段” 等环节, 才能真正形成合力。但这个整合过程 并不容易。一是因为高校的发展已经条块分割, 例如 负责 “管理评估” 和 “教学模式、方法” 的可能是独 立的部门; 二是因为教育本身是一个系统, 要想将目 标和规格的各个要素落实到系统的全过程, 并不是一 件容易的事情; 三是因为很多目标规格在制定的时候 理想的成分多一些, 并没有过多考虑教学的实际过程、 条件、老师的水平, 存在好高鷔远、脱离实际等情况。 尽管所有人都认同“人才培养目标和规格” 的重要性, 但是在实际操作中, 往往存在着目标 “好高憼远” 、 “难以落实”、“缺乏系统性” 等问题。

因此, 要破解以上难题, 就需要以系统化的思维 方式重新审视人才培养目标和规格的概念。制定教学 方案时, 应该紧握培养目标这个核心抓手, 综合规划, 力争将培养目标贯穿于教学的各个环节。基于上述考 虑, 本文借鉴美军 “基于能力的规划 (Capabilitiesbased Planning, 简称 CBP) ” 理论, 以期解决前面提 到的问题。

\section{2. 课程设计的主要模式}

美国著名教育学家 Ralph Tyler（1949）将 “课程 (curriculum) ” 定义为 “教学内容、教学方法和教学 评估”的总和, 是“教学的总体规划” (王海啸, 2004), 即本文所述的教学方案。

在《课程与教学的基本原理》（Basic Principles of Curriculum and Instruction）一书中, Tyler 系统阐 述了构成课程设计的四个环节: 确定教学目标、选择 教育经验、组织教学内容和评价教学结果。其中, 确 定教学目标是课程设计的立足点。由此, 该课程设计 模式被称为 “目标模式”。继承 Tyler 衣钵的 Taba

(Stenhouse, 1975; 张华, 2000) 在《课程开发: 理 论与实践》 (Curriculum Development: Theory and Practice) 一书中, 沿用 Tyler 的直线式课程设计过程, 将 “目标模式” 发展为 8 个步骤: 诊断需求、陈述目 标、选择内容、组织内容、选择教学经验、组织教学 经验、决定评价对象与评价方式、检查平衡性和顺序 性。而后, Wheeler (张华, 2000; 陈珊, 2012) 于 1967 年在《课程过程》 (Curriculum Process) 一书中, 将 直线式 “目标模式” 改为圆环式: 确定目标、选择教 学经验、选择教学内容、组织和统合教学经验及内容、 评价, 以目标为起点, 最终回到目标, 对目标进行调 整, 又开始新一轮课程设计, 如此循环往复。1975 年, 基于对 “目标模式” 的分析与批判, Stenhouse (张华, 2000; 史光孝, 2011; 范敏, 2016) 提出 “过程模式” : 从分析学科结构入手, 选择教学内容, 建立关于学科 的过程、概念与标准等知识形式的课程, 并提供实施 的 “过程原则”。其特点如下: 教学目标是一般性的、 宽泛的, 教学内容立足于课堂上的现实状况, 课程系 统是开放的而非封闭的, 学习评价上强调形成性和发 展性（陈珊，2012）。

“目标模式” 和 “过程模式” 是课程设计中的两 个主要模式, 虽然对课程设计理论影响深远, 但都存 在缺陷。“目标模式”不但注重课程设计流程的模块 化, 而且强调教学目标在整个课程设计过程中的牵引 作用。教学目标是选择教育经验、组织教学内容以及 评价教学结果的准则。例如, 选择教育经验时, 应注 重使学生有机会实践教学目标; 评价教学结果时, 应 检查课程实效与教学目标之间的差距 (杨寒, 2003)。 总体而言, “目标模式” 既含有逻辑性、科学性、系 统性的各个模块, 也有牵引各个模块的教学目标, 这 使得教学内容很明确, 教学活动有效率, 教学结果较 客观（汪霞，2002；史光孝，2011）。虽然 “目标模 式” 在形式上不断完善, 但其本质性缺陷有两点。第 一, 误解了知识的本质, 导致 “教学目标” 形式化 (formalization) 和 工具化 (instrumentalization)

（Stenhouse，1975; 张华, 2000; 范敏, 2016）。为 了达到所谓 “教学目标” , 整个教学过程趋于工业流 程化, 忽略了实际的教育环境, 限制了受教育者的创 造性与主体性。有时所制定的 “教学目标” 没有适当 考虑教学的条件、老师的水平、学生的个性, 存在过 于理想、好高鹗远、脱离实际等情况。第二, 误解了 
知识改进实践的本质，过于强调 “教学目标” 的主导 作用, 割裂了教学经验、教学内容和教学评价等模块 之间的互动（Stenhouse，1975; 张华，2000; 范敏, 2016）。实际上, “很多情况下, 往往是目的与手段 不可分, 目标与内容不可分, 内容与经验不可分” (汪 霞，2002）。这种理论上的模块割裂，在现实中直接 表现为高校各部门的泾渭分明, 甚至分庭抗礼。例如, 负责 “管理评估” 和 “教学模式、方法” 的可能是两 个独立的部门, 在制定教学方案时双方难以协调。 Stenhouse 的“过程模式”则较好地弥补了“目标模式” 的上述缺陷。教学目标和教学内容具有灵活性, 教学 评价不再局限于教学目标的完成程度, 受教育者的主 动性和创造性在动态、开放的教学过程中得到更好地 发展。然而, 这种“过程模式”却又走向另一个极端。 一方面, 由于否定预设的教学目标, 导致课程设计缺 乏计划性和科学性 (陈珊, 2012)。不仅课堂上的教 育者难以把握, 而且教学方案的制定者也无的放矢。 另一方面, 由于制定教学方案时局限于对学科结构的 分析, 忽视了社会需要和学生需要 (史光孝, 2011)。

在两种课程设计模式中, 教学目标的地位或重或 轻, 但这两种模式均未同时对以下问题进行更加细致、 更具操作性的论述: 如何将教学目标落实到教学的全 过程、如何使教学过程形成一个整体系统等等。为解 决上述问题, 首先应该重新审视教学目标 (培养目标 和规格), 与社会需求和个人需求紧密结合, 充实其 内涵; 第二, 不能割裂教学目标与其他教学环节之间 的联系, 应该采用系统化的设计模式; 第三, 在教学 过程中, 不能局限于实现单一教学目标, 而是应该更 加注重培养理解、分析和解决问题等通用能力。鉴于 此, 美军 “基于能力的规划” 框架恰恰可以为解决上 述问题提供思路。

\section{3. “基于能力的规划” 的启示}

所谓 “基于能力的规划” , 是指在考虑各种不确 定性的情况下, 旨在提供能够应对广泛挑战和各种复 杂环境所需的能力, 并在经济框架下在多种建设方案 间进行决策的规划模式 (Capabilities-based Planning is planning, under uncertainty, to provide capabilities suitable for a wide range of modern-day challenges and circumstances while working within an economic framework that necessitates choice）。“基于能力的规 划” 是美国国防部用于武器装备论证的一套框架。该 方法的提出, 是为了解决美军在冷战后面临的 “威胁 不确定性增加”、“决策准确性下降”等一系列问题。

“基于能力的规划” 提供了一套系统界定能力目标、 方法、标准等要素的流程方法。

虽然 “基于能力的规划” 主要为美国、加拿大、 英国、澳大利亚等国的军方所使用, 但是我们认为该 框架的理念和方法对教育领域的教学方案设计有一 定的启示。这主要是因为 “基于能力的规划” 旨在解 决的问题和教学方案设计有诸多相似之处。首先, 军 事领域和教育领域的规划都是涉及诸多因素和环节
的系统工程, 必须统筹规划。其次, 军事领域和教育 领域的规划都受制于主观要求和客观条件的不确定 性。再者, 军事领域和教育领域的规划都需要考虑效 益问题, 确保建设方案能够在尽可能少投入的情况下 获得最大效益。

“基于能力的规划” 框架对教育领域有着重要启 示, 运用此框架, 可从理念和方法上有针对性地解决 上述问题。具体表现在以下三个方面:

（1）以 “全要素” 的理念重新审视 “能力” 概念

如前所述, 能力目标与教学设计、实施和评估等 其它教学环节相脱节是教学设计中普遍存在的痛点。 要解决这一难题, 就不能将能力内涵的界定视作一个 孤立于后续环节的目标制定过程。能力目标固然重要, 但若在实际操作过程中难以实施、无法评估, 则再好 的目标也只能沦为空中楼阁。许多教学方案的初衰很 精彩, 但教师在实施过程中往往感到无所适从。教学 是涉及诸多要素的系统工程, 这就意味着教学设计者 从教学初始阶段就应具备系统思维的能力。“基于能 力的规划”带来的一个重要启示, 就是要以 “全要素” 的理念审视能力问题——不仅要关注目标, 而且要考 虑目标实现的方法、标准、条件、手段和流程等要素。 只有当能力的界定形成一个系统的整体, 才有可能对 教学设计、实施和评估等后续环节发挥更好的引领作 用。

（2）从 “能做事” 的角度界定 “能力” 各要素

教育的一个重要目标, 就是要确保学生毕业后能 够胜任专业对应的工作岗位。因此, 对学生未来可能 从事的工作岗位进行需求分析是教学设计中不可或 缺的一环。然而当前, 相当一部分的需求分析仅聚焦 某一工作情境下毕业生需要 “有什么 (能力素质)”, 很少关注完成某一工作任务需要 “做哪些 (事情)”。 前者的问题在于, 容易忽略某些可能影响工作顺利完 成的关键要素, 导致能力目标的设定与未来工作岗位 的实际情况相错位。根据 “基于能力的规划” 框架, 以 “能做事” 为基本原则, 可以将各项工作逐级拆解 为细微的子任务和分环节。这既使得对工作岗位要求 的把握更加全面, 也使得能力各要素的内涵在同一思 维框架内得到厘清, 从而为整合能力各要素提供抓手。

(3) 将 “可移植” 定为 “能力目标” 的重要原则

“基于能力的规划” 的基本出发点就是从 “基于 威胁” 向 “基于能力” 转变。这一理念和教育领域内 欧美国家提出的核心（通用）技能 (core/generic ski11s）概念不谋而合。无论需求分析环节对专业对 口的工作岗位有多么充分的畅想, 不可否认的是学生 毕业后所面对的仍将是一个充满变数和不确定性的 世界。即使某一看似固定的工作任务, 其要求也可能 会随着条件的变化而发生改变。因此, 在界定教学目 标的过程中, 重要的并不是某些孤立的工作情境对专 业人才的要求, 而应是从众多工作情境中抽象总结出 的一个包含不同维度的能力空间。当学生具备这些核 
心能力，也就具备了应对不同情境的基本素质。“基 于能力的规划”框架恰恰提供了这样一套可用于分析 能力空间的流程和方法。

\section{4. 案例：构建翻译专业硕士教学方案的尝试}

“基于能力的规划” 提供了一套系统决策军事领 域兵力发展方案的流程方法。本节将以翻译专业硕士 的教学方案设计为例, 说明该框架在教育领域的应用 价值。需要说明的是, 教育领域的规划和军事领域的 规划类似, 都需要多部门统筹协调、专业人员反复论 证。作为探索性研究, 本文虽不能展现这一过程的所 有细节, 但会对这一过程进行案例模拟, 以阐明 “基 于能力的规划” 框架在教育领域的可行性。此外, 该 框架在实际操作中, 还有待行政管理环节的介入。

\section{（1）确定人才培养任务}

随着全球化的不断深入和中国国家地位的提升, 中国与世界的联系越来越紧密。经济、政治、军事、 外交等各领域都面临对外交流、宣传、合作、竞争等 方面的机遇和挑战, 亟需大批专业翻译人才从事文秘、 外联、谈判、培训等工作。

\section{（2）设想工作任务场景}

根据第一步的分析结果, 尽可能全面地畅想翻译 专业人才可能面临的工作任务场景。这一想定过程建 立在大规模调查研究基础之上, 需要学校、用人单位 及社会多方专业人员的参与。直于篇幅, 表 1 仅列举 了其中若干种可能的工作任务场景。而在实际规划论 证中, 对工作任务场景的想定要远远超过表 1 的规模 和范围。

\section{表 1 翻译专业硕士可能面临的工作任务场景}

\begin{tabular}{|l|l|}
\hline 1 & 为学术会议做同声传译 \\
\hline 2 & 为商业谈判做交替传译 \\
\hline 3 & 翻译专业学术著作 \\
\hline 4 & 管理团队翻译项目 \\
\hline 5 & 在海外市场进行产品推广 \\
\hline 6 & 为企业建设海外门户网站 \\
\hline 7 & 起草外贸合同 \\
\hline 8 & 从事翻译教学 \\
\hline
\end{tabular}

（3）生成能力想定空间

对具体工作任务场景进行想定, 旨在更直观和准确 地分析翻译专业人才在任职岗位上可能面临的各种挑 战。在表 1 的基础上, 可以抽象凝练出能够用于衡量不 同工作任务场景要求的维度, 它们共同构成了翻译能力 运用的想定空间。表 2 列出了本研究的想定空间的部分 维度。
表 2 翻译能力运用的想定空间维度

\begin{tabular}{|c|c|c|}
\hline & 维度 & 性质 \\
\hline 1 & 外语能力 & 高或低 \\
\hline 2 & 汉语能力 & 高或低 \\
\hline 3 & 信息素养 & 高或低 \\
\hline 4 & 对象国文化 & 熟悉或陌生 \\
\hline 5 & 涉及领域 & 通用领域或专业领域 \\
\hline 6 & 涉及部门 & 单一部门或多部门 \\
\hline 7 & 工作方式 & 独立作业或团体协作 \\
\hline 8 & 工作对象 & 特定人群或普罗大众 \\
\hline 9 & 准备程度 & 突发或预备 \\
\hline
\end{tabular}

构建想定空间, 既需要广泛畅想, 又需要归纳抽 象。广泛畅想是指穷尽一切可能性以界定工作任务场 景的维度要素, 归纳抽象是指在众多维度中梳理总结 出兼具代表性和普适性的维度类别。理想的想定空间, 应可用于界定任何一种工作场景的任务性质和要求。

(4) 制定工作任务目标

在想定空间的基础上，可以更加明确翻译人员在 工作岗位上要完成的工作目标（表 3）。这些目标大 概率预判了翻译人员在具体工作任务中必须解决的 现实问题。

\section{表 3 翻译专业人员的工作目标}

\begin{tabular}{|l|l|}
\hline 1 & 用最短的时间熟悉某一陌生的专业领域 \\
\hline 2 & 用最短的时间熟悉某一陌生的对象国文化 \\
\hline 3 & 迅速识别某一公文格式, 并完成高质量撰写 \\
\hline 4 & 尽快从网络获取某一工作任务的辅助资源 \\
\hline 5 & 与多部门协调某一工作相关事宜 \\
\hline 6 & 组织团队高效完成某一翻译项目 \\
\hline 7 & 向潜在的海外客户介绍某一产品的特性 \\
\hline
\end{tabular}

（5）分解工作任务目标

确定工作任务目标后, 按照 “完成工作任务需要 做什么” 的思路将相对宏观的目标拆解为可以支撑目 标实现的任务清单。任务清单呈现了翻译人员在实际 操作中需要经历和完成的各个步骤。换言之, 翻译人 员按照顺序执行清单中的各项子任务, 就可以保证顺 利完成预定工作任务目标。图 1 所展示的是 “用最短 的时间熟悉某一陌生的专业领域” 这一目标的任务清 单。当然, 同一目标可能有多种实现路径, 不同的规 划者会制定不同的任务清单。在实际操作中被采纳的 任务清单应该是经过多方论证、具有较强可行性的方 案。 


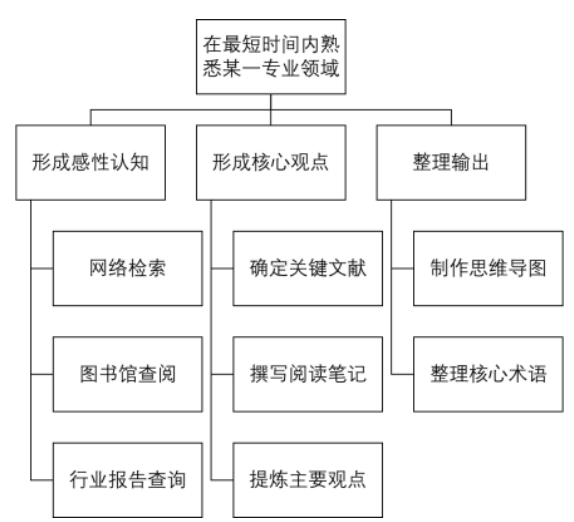

图 1 工作任务目标的分解 (示例)

(6) 确定评估标准

对工作目标进行分解并得到任务清单后, 就要制 定衡量各项子任务是否顺利完成的指标。确定指标, 需要结合步骤 (3) 得到的想定空间的各个维度。例 如, 从表 2 可以看出, 对于翻译专业人员而言, 时间 在很多工作任务场景中都是会影响工作效果的重要 因素。因此，可将 “在多长时间内可以搜集到一定数 量的行业报告” 作为一条评估标准。再如, 从数量的 角度衡量, “一日之内能够阅读多少篇相关文献”也 可成为一条评估标准。又如, “生成的核心术语列表 在所搜集文献中的分布比例” 也可作为一条评估标准。

在这一步得到的评估标准, 很有可能会成为未来 教学设计中的教学目标, 或是形成性评估所采用的总 要参考。评估标准制定得越细致, 越有利于各项教学 目标的考核。

\section{（7）制定教学方案}

能力目标和评估标准得到确认后, 就可以制定相 应的教学方案。教学方案被视为教学手段的系统组合。 各个教学手段都需要针对某一项或某几项能力发展 目标, 同时需要与步骤 (6) 得到的评估标准相挂钩。 教学手段是多元化的, 课程并非唯一选项, 还可以采 取项目设计、课外活动等多种形式。例如, “开设图 书情报检索课程” 可作为针对 “用最短的时间熟悉某 一陌生的专业领域” 这一工作任务目标的教学手段, 但该目标同样可以通过网络在线课程或课外实践项 目的形式实现。但无论采取哪一种教学手段, 都必须 服务于整体的能力发展目标, 并与步骤 (6) 得到的评 估标准相对应。

基于各项工作任务目标得出教学手段列表后, 就 可以对各种手段进行组合, 形成整体教学方案。由于 教学手段多种多样, 它们之间的组合方式也各不相同, 因此整体教学方案可能有多套作为备选。

\section{（8）对教学方案进行评估}

“基于能力的规划” 本质上是一种决策方法, 即 如何在众多方案中挑选最佳方案的方法。在步骤 (7) 的基础上, 对备选的教学方案进行对比评估, 挑选出 效果最好、投入最经济的方案。
效果评价要依据步骤 (6)得到的各项评估标准。 某工作任务目标能否顺利实现, 取决于其下属各子任 务对应的各个评估标准能否顺利实现。这个评价过程 可以采取定量的方法, 最终各套教学方案都有一个对 应的效果指标。

对投入的评价可以基于一套方案所需经费、所需 教员数量、教学管理所需投入等各个指标。同样, 这 个评价过程也可以采取定量的方式, 得到各套方案对 应的投入指标。

在效果和投入均可量化的情况下, 可比较各套方 案的优劣, 从而得到最优的解决方案。

\section{5. 结论}

教学设计最大的难点之一, 就是如何制定教学的 能力目标, 并在教学设计、实施和评估等各环节体现 能力目标对人才培养的总体牵引。若想教学目标贯穿 并体现在整个教学方案的各个环节, 就必须采取系统 的思维方式, 对 “能力” 的内涵进行全面考量。美军

“基于能力的规划” 框架为教育领域解决以上问题提 供了新的思路和解决方案。该框架拓展了能力的内涵, 并将目标、方法、标准、任务等要素融入规划过程的 各个环节, 使其形成一个有机的整体。借鉴该方法, 能够为解决教育领域内 “目标与过程相脱节” 、“手 段与评估不匹配”等一系列常见的问题提供一种新的 解决方案。

需要再一次指出的是, “基于能力的规划” 是一 个涉及诸多部门和环节的复杂过程, 本文仅通过举例 论述该方法在教育领域的应用价值。美军在推行该套 框架的过程中, 也遇到了一些难题。如何更好地认识 这一框架, 吸纳其合理成分, 仍需要进一步深入研究。

\section{项目基金}

本文为湖南省普通高等学校教学改革研 究项目《基于事件域认知模型的涉外军事任务 情境用语研究》(HNJG-2020-0032) 的阶段性成 果之一。

\section{REFERENCES}

[1] Stenhouse, L. An Introduction to Curriculum and Development [M]. London: Heinemann, 1975.

[2] Tyler, R. Basic Principles of Curriculum and Instruction [M]. New York: Harcourt Brace, 1949.

[3] Chen, S. Design and Reform of Specialized Courses for English Major in Chinese Colleges and Universities[D]. Shanghai: Shanghai International Studies University, 2012. 
[4] Diao, W. Reflections on the Research on Teaching Modes[J]. Education Exploration, 2008, (12) : 27-29.

[5] Fan, M. Stenhouse's Comments on the Objectives Model of Curriculum Development[J]. Global Education, 2016, 45 (1) : 56-65.

[6] Liu, M. On the Environment of University Education[M]. Beijing: Aviation Industry Press, 1993.

[7] Pan, M. \& Li, G. Prospects for the Modernization of China's Higher Education in 2030[J]. China Higher Education, 2016, (17) : 4-7.

[8] Shi, G. Study on the Curriculum Design of College English from the Perspective of Hidden Curriculum[D]. Shanghai: Shanghai International Studies University, 2011.

[9] Wang, X. The Objective Model of Curriculum Development and Its Features[J]. Studies in Foreign Education, 2002, (6) : 9-13.

[10] Wang, H. Analysis of the Needs and Conditions in the Curriculum Design of Individualized College English Syllabus[J]. Foreign Languages in China, 2004, (1) : 21-26.

[11] Yang, Q. Study on the "Target Mode" of Curriculum Design and Relevant Issues[J]. Education Science, 2003, 19 (3) : 30-32.

[12] Zhang, H. Curriculum and Teaching Theory[M]. Shanghai: Shanghai Educational Publishing House, 2000.

[13] Zhou, Y. To Enhance Quality Awareness and to Achieve Breakthroughs in Teaching Reform[J]. Journal of Higher Education. 1998, (3) : 5-15.

[14] Zhu, J. To Deepen the Capability-oriented Reform of Talent Cultivation Mode[J]. China Higher Education, 2015, (12) : 35-37. 